\title{
A study about the effects of affective valence on a
}

\section{source-monitoring error: cryptomnesia}

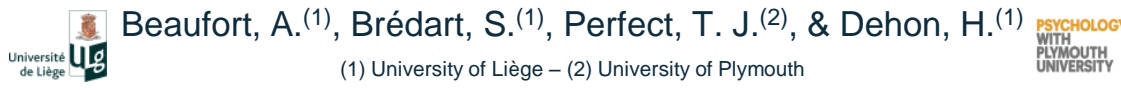

Background The emotional content of the to-be-remembered material could affect source monitoring accuracy as suggested by numerous studies 2,3 . Although inadvertent plagiarism is considered as a source monitoring error and is often linked to creative-emotional environments such as arts, the effect of emotional content on inadvertent plagiarism has never been investigated. Therefore, the objective of our experiment was to examine the possible impact of emotion on inadvertent plagiarism. Inadvertent plagiarism either when a person remembers an item and erroneously thinks that he/she was the generator of that item (RO task) or when the person erroneously thinks that he/she produces the item at the moment although, in fact, this item is a memory not recognized as such (GN task). According to the Paradoxical Negative Emotion hypothesis, negative emotion should capture attention toward central details of a situation and decrease attention toward peripheral details including the source of the encountered information. Consequently, cryptomnesia should increase when the to-be-remembered material is negative. In order to make our experiment as close as possible of the creative processes implicated in reallife, we used the Alternative Uses Task ${ }^{3}$ in a slightly modified version of the Brown and Murphy classical paradigm ${ }^{1}$.

\section{Procedure ${ }^{1,2} \quad$ INITIAL GENERATION (2 participants together)}

"Generate alternately original/non-conventional uses to the presented objects" (1 Positive, 1 Neutral \& 1 Negative object) For each orally generated idea, both participants made 3 judgments:

Valence (-3 "highly negative" $\rightarrow+3$ "highly positive"), Arousal ( 1 "unexciting" $\rightarrow 6$ "very exciting") \& Feasibility ( 1 "unrealizable" $\rightarrow 6$ "feasible")

A one-week delay

RECALL-OWN TASK (2 participants separately)

"Recall as many ideas as you can that YOU personally produced last week." Confidence rating and Remember-Know-Guess judgments

$\longrightarrow(\mathbf{1}=$ "Not sure" to $\mathbf{5}=$ "Sure I said that idea last week")

\section{Participants}

$\mathrm{N}=96$ (48 males) Mean age : 19 years Same-sex dyads

Recall-Own Results Mean plagiarism rate : $7.71 \%$

Percentage RO Responses

\begin{tabular}{lccccccc}
\hline & \multicolumn{2}{c}{ Positive } & \multicolumn{2}{c}{ Neutral } & \multicolumn{2}{c}{ Negative } \\
& Mean & SEM & Mean & SEM & Mean & SEM \\
\cline { 2 - 7 } Correct Response & 64.79 & 1.95 & 62.92 & 2.12 & $<70.63$ & 2.10 \\
Intrusions & 4.58 & 1.04 & 5.63 & 1.05 & 5.00 & 0.88 \\
\hline
\end{tabular}

Percentages of RO plagiarism for each emotional category and gender of participant

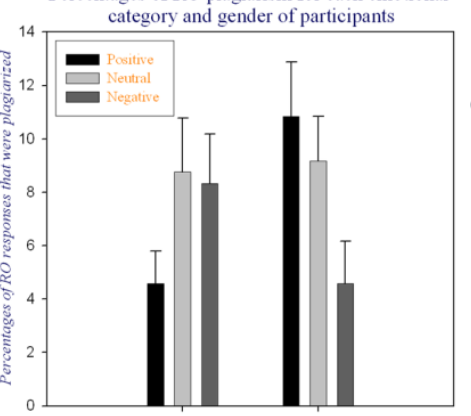

Interaction : Valence*Gender $\left(F_{(2,188)}=5.314, p=0.006, \eta_{p}^{2}=0.054\right)$

Valence Effect : Women only

Negative vs Positive

$\left(F_{(1,94)}=10.1, p=0.002\right)$

Negative vs Neutral

$\left(F_{(1,94)}=3.985, p=0.049\right)$

\section{Confidence ratings}

